\title{
Kink-antikink vortex transfer in periodic-plus-random pinning potential: Theoretical analysis and numerical experiments
}

\author{
W. V. Pogosov, ${ }^{1,2}$ H. J. Zhao, ${ }^{1}$ V. R. Misko, ${ }^{1}$ and F. M. Peeters ${ }^{1}$ \\ ${ }^{1}$ Departement Fysica, Universiteit Antwerpen, Groenenborgerlaan 171, B-2020 Antwerpen, Belgium \\ ${ }^{2}$ Institute for Theoretical and Applied Electrodynamics, Russian Academy of Sciences, Izhorskaya 13, 125412 Moscow, Russia
}

(Received 3 November 2009; published 19 January 2010)

\begin{abstract}
The influence of random pinning on the vortex dynamics in a periodic square potential under an external drive is investigated. Using numerical experiments and theoretical approach, we found several dynamical regimes of vortex motion that are different from the ones for a regular pinning potential. Vortex transfer is controlled by kinks and antikinks, which either pre-exist in the system or appear spontaneously in pairs and then propagate. When kinks and antikinks collide, they annihilate. We provide clear physical interpretations of the observed features.
\end{abstract}

DOI: 10.1103/PhysRevB.81.024513

PACS number(s): 74.25.Uv

\section{INTRODUCTION}

The behavior of an elastic media under the competitive action of a regular potential and disorder is a common problem in various fields of modern physics. Examples of such media are vortex lattices in superconductors ${ }^{1-4}$ or in BoseEinstein condensates of ultracold atoms, ${ }^{5}$ interacting colloids on periodic substrates, ${ }^{6,7}$ charge- and spin-density waves in metals, ${ }^{8}$ polarization density waves in ferroelectrics and many others. Regular pinning potential can be either of artificial origin, as in nanostructured superconductors and in Bose-Einstein condensates with optical lattices, or it can be imposed by the crystal structure of the material. In superconductors, pinning efficiency determines the value of the critical current, while the enhancement of this current is of great practical importance. Theoretical description of such systems is a quite complicated problem, which in one-dimensional case can be reduced to the well-known Frenkel-Kontorova model. ${ }^{89}$ Numerical simulations supplemented by analytical arguments thus can be considered as an effective approach for understanding of basic properties of systems with both regular and random potentials.

Two-dimensional (2D) lattice of repelling particles in the presence of square pinning potential and disorder was recently addressed both theoretically and numerically in Ref. 10 in the context of the flux-line lattices in superconductors. It was demonstrated that in case of weak-disorder pinned vortex lattice is disturbed by specific defects consisting of elastic strings of depinned vortices. These strings intersect and form branched fractal-like clusters, which perhaps can percolate through the system. The phase diagram of such a system turns out to be quite rich, because it is determined by the interplay of three factors: (i) a periodic pinning, (ii) disorder, and (iii) the intervortex repulsion. The third factoreven in the absence of any disorder and at equal concentrations of regular pins and vortices-leads to the appearance of an unconventional state ("half-pinned phase"), when half of vortices are depinned, but the vortex lattice keeps its periodicity. ${ }^{11-14}$

The aim of the present paper is to investigate the vortex dynamics in two-dimensional system in the presence of both a square periodic potential and disorder and to reveal corre- lations between the static defects and the dynamical regimes under an external drive.

The paper is organized as follows. In Sec. II, we present the basic formalism of our model and discuss our moleculardynamics simulation approach. Section III deals with different dynamical regimes of vortex lattice motion. Finally, the conclusions are given in Sec. IV.

\section{MODEL}

We model a three-dimensional superconductor by a $2 \mathrm{D}$ slice assuming vortex cores do not change in the $z$ direction, ${ }^{2,3}$ with regular square array of pinning sites with period $a$ in the presence of additional randomly distributed pins of comparable concentration. Pinning potential, produced by a single site, is modeled by a parabolic function, with $U_{r e g}\left(U_{r a n}\right)$ and $\sigma_{r e g}\left(\sigma_{r a n}\right)$ being the depth and size of potential wells of regular (random) origin; $\sigma_{r e g}, \sigma_{r a n} \ll a$, and $\sigma_{r a n} \sim \sigma_{r e g}$. We will mostly focus on the weak-disorder regime, i.e., when the maximum pinning force by one regular site, $f_{\text {reg }}=2 U_{\text {reg }} / \sigma_{\text {reg }}$, is significantly larger than that of a random site, $f_{r a n}=2 U_{r a n} / \sigma_{r a n}$. We consider the case of the first matching field, i.e., when the number of vortices is equal to the number of regular pins. Vortices are treated within the London approximation, which is accurate for large values of the Ginzburg Landau parameter $\kappa \gg 1$ and low applied magnetic field $H \ll H_{c 2}$. Then the interaction energy of two vortices positioned at $\mathbf{r}_{1}$ and $\mathbf{r}_{2}$ is given by

$$
H_{\text {int }}\left(\mathbf{r}_{1}, \mathbf{r}_{2}\right)=\frac{\Phi_{0}^{2}}{8 \pi^{2} \lambda(T)^{2}} K_{0}\left[\left|\mathbf{r}_{1}-\mathbf{r}_{2}\right| / \lambda(T)\right]
$$

where $K_{0}$ is a modified Bessel function. An important quantity, which is useful for our analytical consideration, is the interaction energy between a regular vortex row and a vortex outside this row. If we place a center of coordinates at one of the vortices in the row, and direct the $y$ axis along the row, then the interaction energy between the vortex row and a vortex located at $(x, y)$ is given by 


$$
H_{\text {int }}^{(\text {row })}(x, y)=\frac{\Phi_{0}^{2}}{8 \pi^{2} \lambda(T)^{2}} \sum_{m=-\infty}^{\infty} K_{0}\left(\frac{\sqrt{x^{2}+(y+m d)^{2}}}{\lambda(T)}\right),
$$

where $d$ is the intervortex distance in the row. By using Fourier transformation for $K_{0}(r)$ and performing a summation in the reciprocal space, Eq. (2) can be rewritten as

$$
\begin{aligned}
H_{\text {int }}^{(\text {row })}(x, y)= & \frac{\Phi_{0}^{2}}{8 \pi \lambda(T) d} \sum_{m=-\infty}^{\infty} \frac{1}{\sqrt{1+\left(\frac{2 \pi m \lambda(T)}{d}\right)^{2}}} \\
& \times \exp \left(-\frac{x}{\lambda(T)} \sqrt{1+\left(\frac{2 \pi m \lambda(T)}{d}\right)^{2}}\right) \cos \frac{2 \pi m y}{d} .
\end{aligned}
$$

Due to the exponent in the right-hand side of this equation, the dependence of $H_{\text {int }}^{(\text {row })}(x, y)$ on $y$ can be very well approximated at low fields [intervortex distances $\sim \lambda(T)$ ] by taking into account only terms with $m= \pm 1$.

To study numerically the motion of vortices we use molecular-dynamics simulations, and we integrate the overdamped equations of motion ${ }^{2,3}$

$$
\eta \mathbf{v}_{i}=\mathbf{f}_{i}=\mathbf{f}_{i}^{v v}+\mathbf{f}_{i}^{v p}+\mathbf{F}_{d}+\mathbf{f}_{i}^{T} .
$$

Here, $\mathbf{f}_{i}$ is the total force acting on vortex $i ; \mathbf{f}_{i}^{v v}$ and $\mathbf{f}_{i}^{v p}$ are the forces due to the vortex-vortex and vortex-pin interactions, respectively, $\mathbf{F}_{d}$ is an external driving force (i.e., a Lorentz force created by an applied current), which is directed along one of the principle axes of the square pinning array; $\mathbf{f}_{i}^{T}$ is the thermal stochastic force. A simulation region contains $20 \times 20$ regular pins, and we use periodic boundary conditions to simulate an infinite array. To explain the results of numerical experiments, we use an analytical approach similar to that of Ref. 10, which is based on the fact that in various configurations most of the vortices remain pinned, so that the vortex transfer occurs through collective defects that can be described reasonably well by just few parameters.

\section{DYNAMICAL REGIMES}

In order to reveal various dynamical regimes in the weakdisorder limit, we carry out numerical experiments and supplement them by the theoretical analysis. The characteristic quantity, which enables us to distinguish between different regimes, is the average vortex velocity $\langle v\rangle$ in the direction of the driving force $\mathbf{F}_{d}$, as a function of $F_{d}=\left|\mathbf{F}_{d}\right|$. Averaging is performed both among all the vortices in the system and over time, after the steady flow is achieved. In general, the smaller the number of depinned and flowing vortices the larger the average vortex velocity. Thus this quantity is similar to the dynamical order parameter that can be defined as a fraction of depinned and moving vortices.

Below we present our results for the following set of parameters: the lattice period for regular pins $a=\lambda(T), \sigma_{\text {reg }}$ $=0.15 a, \sigma_{\text {ran }}=0.2 a, f_{\text {reg }}=0.6 \Phi_{0}^{2} / 8 \pi^{2} \lambda(T)^{3}$, the number of regular and random sites being the same. These results are rather generic with respect to the variation in main parameters, until conditions $\sigma_{r a n}$ and $\sigma_{r e g} \ll a \sim \lambda(T)$ are fulfilled.

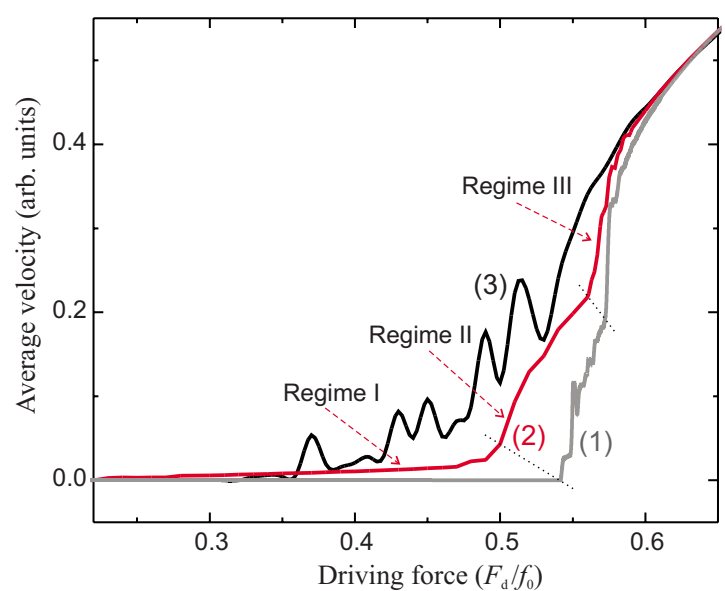

FIG. 1. (Color online) The average vortex velocity as a function of driving for different values of the random pinning force: $f_{\text {ran }}$ $=f_{\text {reg }} / 6$ (curve 1$), f_{\text {reg }} / 3$ (curve 2 ), and $f_{\text {ran }}=f_{\text {reg }} / 2$ (curve 3 ). Black dotted lines show the transitions (crossovers) between different dynamical regimes.

The value of $f_{\text {reg }}$ is chosen in such a way that it allows us to describe the most interesting region of the phase diagram that corresponds to intermediate regular pinning strengths. As shown in Ref. 10, in this regime and for a weak disorder, static defects of pinned square vortex lattice consist of clusters containing elastic chains of depinned vortices. Our simulations show that until we are in the intermediate regime, variations in $f_{\text {reg }}$ do not lead to qualitative changes in the dynamical regimes.

Typical driving dependences of the average vortex velocity are presented in Fig. 1, where one can clearly see three distinct dynamical regions for the case of a weak disorder (curves 1 and 2), while for stronger disorder they are smeared out due to chaotization (curve 3). We associate these different regions with different dynamical regimes of vortex lattice motion. These $\langle v\rangle-F_{d}$ curves of course are sensitive to the particular realization of disorder. However, we found that, in general, the shape of the curve, that is characterized by three different regions, is quite robust with respect to different realizations of disorder, except of some special cases, which are discussed below. The characteristic values of driving force for the crossover regions between different regimes are also rather reproducible, while absolute values of average vortex velocity can vary.

\section{A. Regime-I: Depinning of stripes}

Very weak driving results in no vortex motion (pinned regime). If the driving force $F_{d}$ reaches some threshold value $F_{d}^{(\mathrm{I})}$, part of vortices start to move. An analysis of vortex patterns shows that vortex motion is not individual, since vortices travel collectively in a solitonlike manner, being localized within vortex rows. ${ }^{16}$ Moving collective structures are just depinned kink and antikink defects (stripes) earlier predicted for static configurations. ${ }^{10}$ Such a defect consists of a finite-length chain of depinned vortices, whose length depends on $f_{\text {reg }}$, this length being much larger than $a$ in the regime of intermediate strength of the regular pinning poten- 


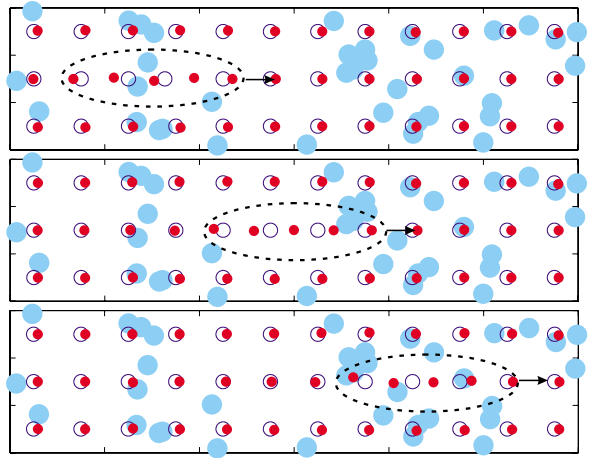

FIG. 2. (Color online) Motion of the stripe shown by three snapshots. Irregular blue (light gray) spots represent positions of random pins, red (dark gray) dots show positions of vortices, and regular black open circles correspond to periodic pins. Dashed lines are guides for eyes indicating positions of defects, arrows show the direction of their motion.

tial. The defects contain either one extra vortex (kink) or one vacancy (antikink). Kinks move in the direction of an applied force, so they represent "compression waves" in vortex rows, as seen in Fig. 2. Vacancy-based antikinks flow in the opposite direction; they can be considered as "decompression waves."

In order to understand why the dynamical threshold is associated with the depinning of kinks, let us first analyze the structure of a static kink. Kink appears due to an additional vortex in a particular region of the system. ${ }^{10}$ Because of the intervortex repulsion, it displaces other vortices within one row from their pinned positions, while vortices in all other rows remain pinned, i.e., one-dimensional deformation field of a vortex chain changes by $a$ along the kink. In the limit of narrow regular pins, $\sigma_{\text {reg }} \ll a$, one can assume that vortices in a kink are completely depinned, whereas all other vortices in the same row are pinned, as well as vortices in other rows. The total energy of the kink compared to the unperturbed system is thus given by the sum of three contributions, $E_{1}$, $E_{2}$, and $E_{3}$. The first term, $E_{1}$, is due to the energy gained by each of $D$ depinned vortices. Before the depinning, each of these vortices was located in the potential well of the depth $U_{\text {reg }}=f_{\text {reg }} \sigma_{\text {reg }} / 2$. After the depinning, its energy became nearly equal to the average regular pinning energy within the row, $-\frac{4 \sigma_{\text {reg }}}{3 a} U_{\text {reg }}$. Thus, $E_{1}$ is given by

$$
E_{1} \approx \frac{f_{r e g} \sigma_{r e g}}{2}\left(1-\frac{4 \sigma_{r e g}}{3 a}\right) D .
$$

The second term, $E_{2}$, is an energy decrease due to the interaction with vortices from the surrounding pinned vortex rows. If we are far enough from the threshold of the transition to the half-pinned phase, ${ }^{11-14}$ i.e., in the intermediate regime of pinning strength, this contribution is sufficiently smaller than $E_{1} \cdot{ }^{10}$ Therefore, in the leading order, we can assume that positions of depinned vortices in the row are not correlated with the minima of the potential interaction with surrounding rows, so that the term $E_{2}$ can be easily found from Eq. (3) by averaging over these positions

$$
E_{2} \approx-\frac{\Phi_{0}^{2}}{8 \pi^{2} \lambda(T)^{2}} e^{-2 \pi} D .
$$

The third contribution, $E_{3}$, is associated with the increase in the vortex-vortex interaction energy within the row, where the kink is localized, this increase being caused by one excess vortex. Under assumptions of the elasticity theory, one can take into account only interaction between nearest neighbors in the row, from which it follows that distances $l$ between nearest vortices inside the kink are all the same, $l$ $=D a /(D+1)$. By summing the energies of the vortex-vortex interaction, we obtain a $D$-dependent part $E_{3}$ of the corresponding contribution to the energy of the kink, which, in the leading order in $a / D$, is given by

$$
E_{3}=\frac{\Phi_{0}^{2}}{8 \pi^{2} \lambda(T)^{3}} K_{1}[a / \lambda(T)] \frac{a}{D} .
$$

The optimal number of vortices in the kink $D$ now can be found by the minimization of $E_{1}+E_{2}+E_{3}$

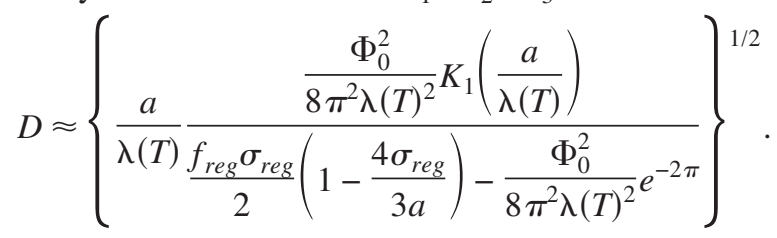

For the used values of parameters, we obtain $D \approx 4-5$, while in numerical simulation we found that $D \approx 5-6$, so that our analytical approach provides a quite good estimate.

If we now suddenly turn on an applied force $F_{d}$ acting on each vortex, the net external force acting on the whole kink is $D F_{d}$. At the same time, the force of resistance is due to the fact that the first and last vortices in front and behind the kink are pinned, and thus the maximum resistance force is $2 f_{\text {reg. }}$. By equating the external force to the force of resistance, we obtain a simple condition for the depinning of the kink

$$
F_{d}^{(\mathrm{I})} \approx \frac{2 f_{r e g}}{D} .
$$

Decoupling of kinks from the underlying pinning array signals as an increase in the average vortex velocity, which is used here as an indicator for distinguishing between different dynamical regimes. In experiments, such a decoupling would result in the increase in sample's resistance, since vortex motion in superconductors is associated with dissipation. Notice that Eq. (9) can be also obtained by considering a balance of the forces, acting on each vortex in the kink, one vortex in front of the kink, and one vortex behind it, and then by summing up these forces. An alternative approach is to use a continuum approximation for the one-dimensional FrenkelKontorova model, ${ }^{8}$ from which one can deduce a deformation field for the vortex chain inside the kink caused by external driving and then equate to $f_{r e g}$ the pressure this row exposes on the first or the last pinned vortex along the kink. Equation (9) demonstrates that, in the large $D$ limit, the stripe behaves nearly as a rigid body containing $D$ particles. This is the explanation why it is depinned at relatively low 
driving forces, i.e., significantly smaller than $f_{\text {reg. }}$. This rigidity is linked to the fact that regular potential is weak and that is why kink is much longer than $a$ : it is favorable that an excess vortex depins many other vortices in the row. Therefore, vortex chain deformations under an external drive remain small until kink is pinned, resulting in nearly periodic locations of vortices inside it. Equation (9) implies that $F_{d}^{(\mathrm{I})}$ is very sensitive to the kink's length, which, in turn, depends on the regular pinning potential. The tendency is that, the stronger regular pinning the shorter kinks and antikinks, so that in the limit of strong pinning one reaches the regime when defects are no longer collective. ${ }^{10}$ The analysis of this limit, i.e., when the vortex-vortex interaction does not play an important role anymore, is beyond the scope of the present work.

The results obtained by using Eqs. (8) and (9) are in a good semiquantitative agreement with results of our numerical simulations: depinning of stripes is predicted to occur at $F_{d}^{(\mathrm{I})} \approx 0.48 f_{\text {reg }}$ for the set of parameters we used, while in the numerical simulations this value was ranged between $0.30 f_{\text {reg }}$ and $0.42 f_{\text {reg }}$, for a weak random pinning strength and in different trials. Note that in the particular initial configuration shown in Fig. 1 by curve 1, there was no preexisted stripe in the simulation region, which is reflected by the absence of any current up to the dynamical regime-II. This is an example of how different realizations of disorder in our finite-size simulations can lead to different results. The smaller the system the higher the probability that there are no pre-existed kinks. However, in the infinite system these defects have a finite concentration and therefore regime-I always exists. In general, the main requirement for the determination of dynamical regimes in the studied system is that the simulation region has to be significantly larger than the length of a single kink/antikink, since the dynamics is controlled by kinks and antikinks. This condition is justified in our simulations.

In our numerical experiments, we found that kinks and antikinks always disappear when they collide. A similar process, known as kink-antikink annihilation, was studied in Ref. 15 within the sine-Gordon model applied for spatially inhomogeneous media with dissipation and under an external drive, i.e., essentially the conditions realized in our simulations. Another remarkable but quite rare process, in a weakdisorder regime, is kink sticking by bunches or voids of random pins. These two processes lead to a decay in time of the total current. Figure 3 shows typical time dependences of the current, where two processes are addressed: (i) when kinks and antikinks annihilate (curve 1) and (ii) when they persist (curve $1^{\prime}$ ). It is obvious, however, that in infinite systems all the kinks and antikinks have to disappear, since the total number of kinks and antikinks in the single row is the same. Nonvanishing and stable motion in the weak-disorder regime thus appears as an artifact of a finite-size simulation region with periodic boundary conditions.

Although the vortex transfer is associated with the depinning of pre-existed kinks and antikinks, static defects in the weak- and intermediate-disorder regimes consist not only of these defects. Kinks and antikinks can be just parts of clusters. ${ }^{10}$ One can naively expect that these clusters act as easy channels for the vortex transfer. Instead, low driving

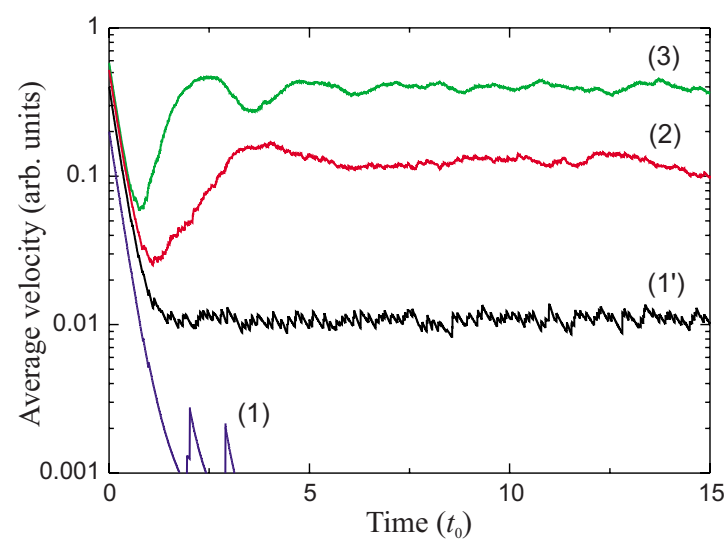

FIG. 3. (Color online) Time dependence of the average vortex velocity for different dynamical regimes at $f_{\text {ran }}=0.2$. Here $t_{0}$ $=4 \pi^{2} \lambda(T)^{4} \eta / \Phi_{0}^{2}$. Curves 1 and $1^{\prime}$ correspond to regime I at $F_{d}$ $=0.4$ and 0.2 , respectively. Curves 2 and 3 show regimes II and III for $F_{d}=0.52$ and 0.57 , respectively.

partially "heals" such defects. The reason is that clusters basically consist of two types of segments. Segments of the first kind contain no vacancies or excess vortices, in contrast with segments of the second type, which are nothing but stripelike defects. For low driving, vortices inside segments of the first kind move collectively to their nearest vacant pins. Such a delicate healing, however, is not possible for segments of the second type, which contain excess vortices or vacancies. Therefore, stripes do persist in the sample, while low driving leads to the fragmentation of clusters. A typical driving force, which heals such defects, can be estimated by considering the limiting case of an infinite chain of depinned vortices. An effective pinning force for vortices from the chain is created by interactions with surrounding rows of pinned vortices. ${ }^{10}$ From Eq. (3), it is straightforward to obtain the following estimate:

$$
F_{d}^{(h)} \approx \frac{\Phi_{0}^{2}}{4 \pi \lambda(T)^{2} a} e^{-2 \pi} .
$$

In the regime of intermediate regular pinning strength, $F_{d}^{(h)}$ is much smaller than $F_{d}^{(\mathrm{I})}$. We will show, however, that $F_{d}^{(h)}$ plays an important role in the dynamical regimes appearing for higher $F_{d}$. Note that the effect of re-entrance of square pinned lattice under a weak external drive was revealed in Ref. 12 for the half-pinned phase (in the absence of disorder). This feature is consistent with the present results, since chains of depinned vortices are nothing but nuclei of this phase. $^{10}$

As mentioned above, here we consider only the case of the first matching field, when the density of vortices and regular pins are exactly the same. It is, however, clear that small differences between these densities would lead to the appearance of additional kinks or antikinks in the static configuration, such that their numbers will be also different from each other. From that, one can conclude that regime-I (as well as other regimes) will be preserved, although motion of kinks and antikinks will be nonvanishing. 

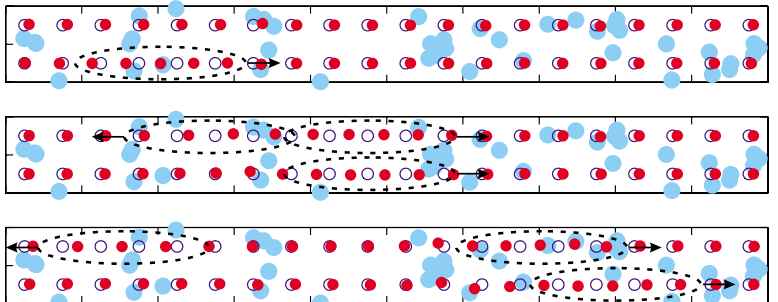

FIG. 4. (Color online) The same as Fig. 1 but with generation of kink-antikink pair by moving stripe, shown by snapshots. Initially kink flows in the bottom row, then it creates a kink-antikink pair in the upper row.

\section{B. Regime-II: Generation of kink-antikink pairs}

Now we discuss the origin of the second cusp in curves 1 and 2 of Fig. 1, which corresponds to the dynamical regimeII. We found by numerical simulations that, when driving approaches some critical value $F_{d}^{(\text {II) }}$, kink-antikink pairs appear spontaneously from time to time. ${ }^{16}$ New kinks move in the direction of the drive, whereas antikinks propagate in the opposite direction. Because of the intensive generations of new kinks and antikinks, the average current increases significantly compared to regime-I, as seen from Fig. 3. Most of the pair generation events are triggered by already excited and moving stripes, which create an additional washboard drive acting on the adjacent rows. Therefore, new kinkantikink pairs appear in the rows adjacent to the row with a moving kink, as shown in Fig. 4 by three snapshots: one of the vortices is depinned, then it creates an area of vortex row compression in front and the area of decompression behind, these two areas being transformed into the kink-antikink pair. It is easy to realize that the amplitude of this additional periodic drive is equal to $F_{d}^{(h)}$ in the limit of long kinks $(D \gg 1)$, so the total effective driving in the row, adjacent to the moving stripe, is $F_{d}^{(e f f)} \approx F_{d}+F_{d}^{(h)}$. However, in some trials there were no pre-existed kinks within a simulation region (see, for instance, curve 1 in Fig. 1), but kink-antikink pairs did appear by themselves in some "weak points," where vortices were additionally strongly displaced in the direction of the drive by random pins. Therefore, we conclude that, in regime-II disorder is the key factor of kinkantikink nucleation, while moving defects can assist to this process. Besides, the comparison with the numerical results shows that the value of $F_{d}^{(h)}$ is too low to explain the data for $F_{d}^{(\mathrm{II})}$ by only a washboard potential of moving stripes.

Let us now analyze how disorder can lead to the generation of kink-antikink pairs and estimate a minimum value of the driving force $F_{d}^{(\mathrm{II})}$ that triggers this process. We place a center of coordinates in one of the regular pinning sites with axis $x$ being directed along the driving force. In the absence of disorder, this driving force shifts the pinned vortex to the point with coordinates $\left(r_{0}, 0\right)$, where $r_{0}=\sigma_{\text {reg }} F_{d} / f_{\text {reg }}$. Moving kink or antikink in the adjacent rows leads to an additional displacement and the total amplitude of the displacement can be estimated by changing $F_{d}$ to $F_{d}^{(e f f)}$. If we now take into account a disorder and neglect the vortex-vortex interaction within the row, random force in the point $\left(r_{0}, 0\right)$ must be nonzero in order to depin the vortex. This restricts possible locations of the random pin(s) acting on the vortex at $\left(r_{0}, 0\right)$ : there should be at least one pin accommodated within the circle of radius $\sigma_{\text {ran }}$ with the center at $\left(r_{0}, 0\right)$. Next, we assume that random force acting on this vortex is created by only one random pinning site, i.e., we neglect possible overlaps between random pins. This is a reasonable assumption (in the leading order in $\sigma_{r a n} / a$ ) provided that $\sigma_{r a n} \ll a$ and concentration of random pins is not very high, $n_{\text {ran }} \sim 1 / a^{2}$. The optimal position for the pin, which minimizes $F_{d}^{(I I)}$, is in the $x$ axis at $\left(\sigma_{r a n}+r_{0}, 0\right)$. This condition implies that two criteria are satisfied: (i) the random force is nonzero at $\left(r_{0}, 0\right)$ and it is directed along the driving force and (ii) at the edge of the regular pin, $\left(\sigma_{\text {reg }}, 0\right)$, where the regular pinning force is maximum, the opposite random force, $f_{\text {ran }}\left(\sigma_{r a n}+r_{0}\right.$ $\left.-\sigma_{r e g}\right) / \sigma_{r a n}$, is also maximum, provided that the criterion (i) is satisfied. This force together with the driving force $F_{d}^{(e f f)}$ should compensate $f_{\text {reg }}$ at the edge of the regular pin. From this we find the critical driving force $F_{d}^{(\text {II) }}$ that depins a given vortex

$$
F_{d}^{(\mathrm{II})} \approx \frac{f_{\text {reg }}-f_{\text {ran }}\left(1-\frac{\sigma_{\text {reg }}}{\sigma_{\text {ran }}}\right)}{1+\frac{f_{\text {ran }}}{f_{\text {reg }}} \frac{\sigma_{\text {reg }}}{\sigma_{\text {ran }}}}-F_{d}^{(h)} .
$$

Starting from $F_{d}=F_{d}^{(\mathrm{II})}$ one can find an area in the vicinity of each vortex, where a random pin can be positioned, such that it will depin this vortex (with the assistance of moving stripe in one of the two adjacent rows). In an infinite system for a finite concentration of random pins, the concentration of such weak points is also finite. Starting from higher drivings, $F_{d} \approx F_{d}^{(\mathrm{II})}+F_{d}^{(h)}$, kink-antikink pairs can be generated in weak points without an assistance of moving defects that agrees with the results of our numerical experiments. When deriving Eq. (11), we did not take into account the mutual repulsion of vortices within the row, which acts against the depinning of a particular vortex. According to our estimates, such a resistance force is too small to noticeably change $F_{d}^{(\mathrm{II})}$, since $\sigma_{r e g}-r_{0} \ll \sigma_{r e g}$. Equation (11) is in a reasonably good agreement with our numerical results for a weak disorder. For instance, according to this equation, $F_{d}^{(\text {II) }}$ corresponding to curves 1 and 2 in Fig. 1 should be around $0.85 f_{\text {reg }}$ and $0.71 f_{\text {reg }}$, respectively, while in numerical experiments these quantities are around $0.89 f_{\text {reg }}$ and $0.78 f_{\text {reg }}$. Variation in these quantities from realization to realization of disorder was within few percent (provided that presence or absence of pre-existed stripes was taken into account properly, when analyzing the data), except of some special cases. Among them, the most frequent was the situation when the number of pre-existed kinks inside the simulation region was much higher than the average one, so that together they were able to produce locally larger washboard potential, which resulted in smearing out the dynamical regimes.

In the limit of a very weak disorder, Eq. (11) reduces to $F_{d}^{(\mathrm{II})} \approx f_{\text {reg }}-f_{\text {ran }}-F_{d}^{(h)}$, which clearly shows a competition between regularity and disorder, the latter factor being enhances by moving kinks through an additional term $F_{d}^{(h)}$. This condition is not sensitive to the particular form of the potential created by a single site, this fact reflecting the universal- 
ity of the obtained criterion. Using similar geometrical arguments (but in two dimensions), we also found that above $F_{d}^{(\mathrm{II})}$, concentration of weak points grows as $\sim\left(F_{d}-F_{d}^{(\mathrm{II})}\right)^{3 / 2}$, so that the generation of kink-antikink pairs is intensified.

We also notice that, due to a continuous generation of kink-antikink pairs, vortex motion within regime-II no longer decays in time, unlike for regime-I. The motion occurs via groups of kinks and antikinks traveling in the opposite directions and creating, from time to time, new defects. One of these defects joins the same group, whereas its counterpartner starts to flow in the opposite direction. In a steadyflow regime, replication of defects has to be balanced with their annihilation, under collisions of individual kinks and antikinks or their groups.

\section{Regime-III: Free proliferation of kink-antikink pairs}

We finally revealed third dynamical regime, which appears as the last region in curves 1 and 2 in Fig. 1, at drivings, which are already very close to the regular pinning force $f_{\text {reg. }}$. In this regime, washboard drive produced by moving kink, is already strong enough to generate kink-antikink pairs freely without an assistance of the disorder. The corresponding driving is then simply given by

$$
F_{d}^{(\mathrm{III})} \approx f_{r e g}-F_{d}^{(h)} .
$$

In this regime, kink-antikink pairs start to proliferate very intensively being triggered by the motion of defects: preexisted kinks immediately generate kinks and antikinks; in turn, these defects create new pairs, and so on. ${ }^{16}$ The area of vortex motion rapidly extends until it covers the whole simulation region. In regime-III, no "islands" are found, where domains of vortex lattice can be pinned for a long time. According to Eq. (12), $F_{d}^{\text {(III) }}$ is around $0.98 f_{\text {reg }}$, and this value is also in a reasonably good agreement with the numerical results, shown in Fig. 1 (approximately, $0.96 f_{\text {reg }}$ for curve 1 and $0.94 f_{\text {reg }}$ for curve 2 , these results typically being reproducible within several percents).

Let us now briefly discuss the dynamics of vortices, when the disorder is no longer very weak, so that it not only triggers motion of defects but also significantly affects it. The general tendency is that disorder smears out well-separated dynamical regimes, as seen from curve 3 in Fig. 1. Kinks and antikinks now can easily bend and jump from row to row. However, very surprisingly, solitonlike origin of the vortex transfer is extremely robust against the disorder, up to the regime of strong disorder, $f_{\text {ran }} \sim f_{\text {reg }}$. In the limit of the very strong disorder, $f_{\text {ran }} \gg f_{\text {reg }}$, vortex flow is localized in narrow streams, in which vortices flow one by one. Another mechanism of vortex transfer is pumping, when vortices are pushed into "traps," until their mutual repulsion breaks the blockade.

Note that here we analyzed the first-matching-field regime, while a little imbalance between the numbers of regular pins and vortices could serve as an additional source of disorder. As was shown in Refs. 2 and 3, this imbalance results in different dynamical regimes including vortex flow in "incommensurate rows" and negative-differentialresistivity (NDR) parts of the VI curve of $\mathrm{N}$ type $\mathrm{e}^{2,3}$ and $\mathrm{S}$ type. ${ }^{3}$ Very recently, the first experimental observation of the N-type NDR phase has been reported. ${ }^{17}$ (See also a related experiment ${ }^{18}$ on a triangular array of pins, where channeling of vortices can be suppressed by the random removal of pinning sites. ${ }^{19}$ )

\section{CONCLUSIONS}

We studied the competitive effect of periodic square and weak random pinning potentials on the dynamics of vortices in two dimensions. We found new dynamical regimes, which display themselves through distinct regions on the curves of average vortex velocity versus external driving force. There are three regimes, in which vortices move in a solitonlike collective structures traveling within individual vortex rows. These are kinks, each containing an excess vortex and moving in the direction of an external drive, and antikinks, flowing in the opposite direction and containing a vacancy. When colliding, kinks and antikinks annihilate. In the first regime, pre-existed static kinks and antikinks decouple from the underlying pinning array and propagate in the system. In the second regime, moving defects excite secondary kinkantikink pairs in the adjacent rows in certain weak points, which are more corrupted by disorder. In the third regime, these pairs are excited by moving kinks and antikinks freely, not only in weak points, due to their additional washboard potential. We presented analytical semiquantitative estimates for characteristic values of driving forces leading to crossovers between the regimes, which are in a good agreement with our numerical results.

Although we have concentrated on vortices in superconductors, it is clear that similar dynamical regimes will be realized in other two-dimensional systems with square-lattice potentials, containing repelling particles. Moreover, disorderinduced kink-antikink generation under an external drive can appear as a rather universal phenomenon, which exists for systems and lattice potentials of various dimensionalities.

\section{ACKNOWLEDGMENTS}

This work was supported by the "Odysseus" Program of the Flemish government, FWO-Vl, and IAP. W.V.P. acknowledges support from RFBR (Contract No. 09-02-00248).

\footnotetext{
${ }^{1}$ M. Baert, V. V. Metlushko, R. Jonckheere, V. V. Moshchalkov, and Y. Bruynseraede, Phys. Rev. Lett. 74, 3269 (1995).

${ }^{2}$ C. Reichhardt, C. J. Olson, and F. Nori, Phys. Rev. Lett. 78, 2648 (1997); Phys. Rev. B 58, 6534 (1998).
}

\footnotetext{
${ }^{3}$ V. R. Misko, S. Savel'ev, A. L. Rakhmanov, and F. Nori, Phys. Rev. Lett. 96, 127004 (2006); Phys. Rev. B 75, 024509 (2007).

${ }^{4}$ G. R. Berdiyorov, M. V. Milošević, and F. M. Peeters, Phys. Rev. B 76, 134508 (2007).
} 
${ }^{5}$ S. Tung, V. Schweikhard, and E. A. Cornell, Phys. Rev. Lett. 97, 240402 (2006).

${ }^{6}$ A. Libál, C. Reichhardt, and C. J. Olson Reichhardt, Phys. Rev. Lett. 97, 228302 (2006).

${ }^{7}$ D. Deb and H. H. von Grunberg, J. Phys.: Condens. Matter 20, 245104 (2008).

${ }^{8}$ P. M. Chaikin and T. C. Lubensky, Principles of Condensed Matter Physics (Cambridge University Press, Cambridge, 1995).

${ }^{9}$ O. M. Braun and Y. S. Kivshar, The Frenkel-Kontorova Model (Springer, Cambridge, 2004).

${ }^{10}$ W. V. Pogosov, V. R. Misko, H. J. Zhao, and F. M. Peeters, Phys. Rev. B 79, 014504 (2009).

${ }^{11}$ V. N. Rudko, O. N. Shevtsova, and S. V. Shiyanovsky, Fiz. Nizk. Temp. 22, 1314 (1996).

${ }^{12}$ W. V. Pogosov, A. L. Rakhmanov, and V. V. Moshchalkov, Phys. Rev. B 67, 014532 (2003).

${ }^{13}$ J. W. Reijnders and R. A. Duine, Phys. Rev. Lett. 93, 060401
(2004).

${ }^{14}$ H. Pu, L. O. Baksmaty, S. Yi, and N. P. Bigelow, Phys. Rev. Lett. 94, 190401 (2005).

${ }^{15}$ V. V. Konotop and L. Vazquez, Nonlinear Random Waves (World Scientific, Singapore, 1994).

${ }^{16}$ See supplementary material at http://link.aps.org/supplemental/ 10.1103/PhysRevB.81.024513 for mov. (1) regime-I: motion of individual stripes, pre-existed in the system; mov. (2) regime-II: generation of kink-antikink pairs by simultaneous effect of disorder and moving defects; and mov. (3) regime-III: free generation of kink-antikink pairs by moving defects.

${ }^{17}$ J. Gutierrez, A. V. Silhanek, J. Van de Vondel, W. Gillijns, and V. V. Moshchalkov, Phys. Rev. B 80, 140514(R) (2009).

${ }^{18}$ M. Kemmler, D. Bothner, K. Ilin, M. Siegel, R. Kleiner, and D. Koelle, Phys. Rev. B 79, 184509 (2009)

${ }^{19}$ C. Reichhardt and C. J. Olson Reichhardt, Phys. Rev. B 76, 094512 (2007) 